\title{
Correction
}

\section{Correction: Dougherty et al., The Disruption of Celf6, a Gene Identified by Translational Profiling of Serotonergic Neurons, Results in Autism-Related Behaviors}

In the article "The Disruption of Celf6, a Gene Identified by Translational Profiling of Serotonergic Neurons, Results in Autism-Related Behaviors” by Joseph D. Dougherty, Susan E. Maloney, David F. Wozniak, Michael A. Rieger, Lisa Sonnenblick, Giovanni Coppola, Nathaniel G. Mahieu, Juliet Zhang, Jinlu Cai, Gary J. Patti, Brett S. Abrahams, Daniel H. Geschwind, and Nathaniel Heintz, which appeared on pages 2732-2753 of the February 13, 2013 issue, a sentence in the legend for Figure 10 should read "Data are plotted as a percentage of WT ion count levels" rather than "Data are plotted as a percentage of WT levels." Statistical results are the same whether ion counts (plots) or pmols (table) of the extracted samples are used in the analysis. This correction does not affect data interpretation or the conclusions of the paper. The legend for Figure 10 has been corrected on the online PDF version.

DOI: $10.1523 / J N E U R O S C I .2895-13.2013$ 